\title{
DESIGN, SIMULATION AND TEST OF PULSED PANOFSKY QUADRUPOLES
}

\author{
Y. Li ${ }^{\#}$, P.Chin, R. Kishek, M.Reiser, M. Venturini, J.G. Wang a and Y. Zou, \\ University of Maryland, College Park, MD 20742 \\ T.F. Godlove, FM Technologies, Inc. Fairfax, VA 22032
}

\begin{abstract}
Two Panofsky quadrupoles with rectangular aperture and fast rise time are proposed for the injection area in the University of Maryland electron ring project (UMER) $[1,2,3]$. The theoretical current distribution needed on the surface of a rectangular aperture to produce a quadrupole field is derived [4]. The conductor location is determined mostly by the theoretical current distribution, with some free factors to optimise the field. The design is based on the linearity of longitudinal integrated field. Each quadrant of the Panofsky quadrupole consists of 10 loops of conductors to minimize inductance while retaining the field quality. A 2:1 scaled model has been made and the magnetic field was measured. Two 1:1 models have been made to measure inductance, mutual inductance and the rise time. Simulations of beam propagation with Panofsky quadrupoles demonstrated the linearity of the design.
\end{abstract}

\section{INTRODUCTION}

An electron ring, UMER, is under development at the University of Maryland for the purpose of studying space charge dominated beams in a circular FODO lattice $[1,2]$. Two pulsed Panofsky quadrupoles shown in Figure 1 with rectangular aperture and fast rise time are proposed in the injection area [3]. Q1 is centered on the ring, replacing one of the regular ring quadrupoles. It must be turned on after the injection beam passes by. Q2 is centered on the injection line and is on only during injection. The dimensions of Q1 and Q2 are slightly different so that they can be overlapped physically.

In the design, due to the small aspect ratio, the integral of the field along the $\mathrm{z}$-direction, rather than the field itself is used. The magnetic field is calculated with a computer code MAG-PC from certain conductor locations. The goal of our design is:

- To have good enough field linearity. Inside 0.7R ( $\mathrm{R}$ is the pipe radius), the linearity should be better than $1 \%$. The integral gradient is 28 Gauss.

- To have fast enough time constant. The field construction time should be less than 100 150ns for $50 \mathrm{~ns}$ bunch. Small inductance is preferred.

"Work supported by the US Department of Energy.

${ }^{*}$ Email: 1iyun@glue.umd.edu

a: Present Address: ORNL and Visiting at BNL.
- To have as small as possible voltage and power requirement to the pulse generator.

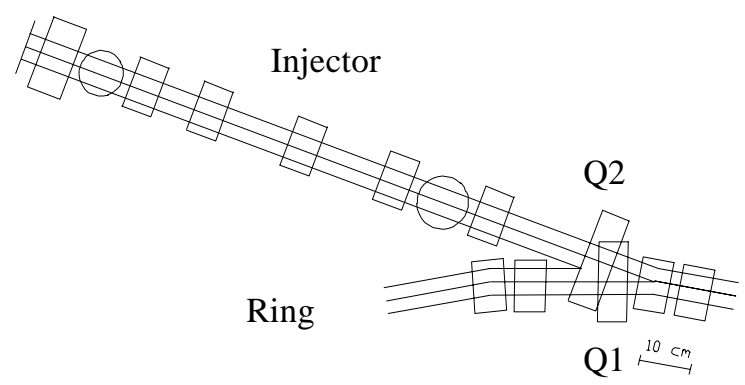

Figure 1: Schematic injector layout of UMER.

\section{DESIGN}

The conductors are located on the four surfaces of a rectangular box. At first two designs, a parallel structure and a loop structure, were explored. In the parallel structure design, the active conductors with same length are all parallel with the $\mathrm{z}$-axis. In the loop structure design, the conductors form loops in each of the quadrants. After carefully designing and comparing, the loop structure was chosen because it needs less current and voltage while both the designs satisfy the field linearity and rise time requirement. Only the loop structure is discussed in the later sections of this paper.

\subsection{Theoretical Formulas}

The surface current distribution for a quadrupole with a rectangular aperture is derived [4]. The current distribution is such that all the integrated (along the $\mathrm{z}$ axis) multipole components of the field vanish, except for the quadrupole term. The surface current density along the z-axis on the top and bottom surface, $\rho_{z}(x)$, and that on the left and right surface, $\rho_{z}(y)$ are:

$$
\begin{aligned}
& \rho_{z}(x)=\sum_{l=0}^{l \max } \rho_{l}\left(1+\frac{x^{2}}{a^{2}}\right)^{-1 / 2}\left(\left(1+i \frac{x}{a}\right)^{l}+\left(1-i \frac{x}{a}\right)^{l}\right) \\
& \rho_{z}(y)=\sum_{l=0}^{l \max } \rho_{k}\left(1+\frac{y^{2}}{b^{2}}\right)^{-1 / 2}\left(\left(1+i \frac{y}{b}\right)^{k}+\left(1-i \frac{y}{b}\right)^{k}\right)
\end{aligned}
$$

where $x, y$ are the horizontal and vertical axis and $a, b$ are the half length of the rectangular aperture in the $\mathrm{x}$ and $y$ direction. The current density is then approximated by the first two terms: $\rho_{z}(x)=\alpha \cdot \rho_{0}(x)+(1-\alpha) \cdot \rho_{1}(x)$, with 
$\alpha$ the weight of the first term. Similar in $y$ with the weight $\beta$.

In the loop structure with a continuous current model, the active currents (currents that go along the z-axis) do not have the same length because of the return current which goes in the $\mathrm{x}$ or $\mathrm{y}$ direction. Let the effective active current (adjusted by the current length) to be the current needed to produce a quadrupole field. Then making use of the continuity of the current [5], the curve of the loop corner must satisfy:

$$
\begin{aligned}
& \left(\frac{f(x)}{L}\right)^{2}=1-\alpha\left(\frac{x}{a}\right)-\left(\frac{1-\alpha}{k}\right) \operatorname{Arcsinh}\left(\frac{x}{a}\right), \\
& \left(\frac{g(y)}{L}\right)^{2}=1-\beta\left(\frac{y}{b}\right)-\left(\frac{1-\beta}{k}\right) \operatorname{Arcsinh}\left(\frac{y}{b}\right)
\end{aligned}
$$

where $k=\operatorname{Arc} \sinh (1)$ is a constant; $L$ is half length of the quadrupole.

\subsection{Conductor Location}

The return current, which is perpendicular to the z-axis, is located with equal intervals at $Z[n]$. The active conductors are then located according to the curve of the loop corners. Their location, $X[n]$ and $Y[n]$, must satisfy:

$$
\begin{aligned}
& \left(\frac{Z[n]}{L}\right)^{2}=1-\alpha\left(\frac{X[n]}{a}\right)-\left(\frac{1-\alpha}{k}\right) \operatorname{Arcsinh}\left(\frac{X[n]}{a}\right), \\
& \left(\frac{Z[n]}{L}\right)^{2}=1-\beta\left(\frac{Y[n]}{b}\right)-\left(\frac{1-\beta}{k}\right) \operatorname{Arcsinh}\left(\frac{Y[n]}{b}\right)
\end{aligned}
$$

The factors $\alpha$ and $\beta$ are adjusted carefully to optimise the field and keep the distance between the conductors not too small. The number of loops $\mathrm{N}$ is a trade-off between the field quality and the inductance of the circuit.

Top and bottom surface

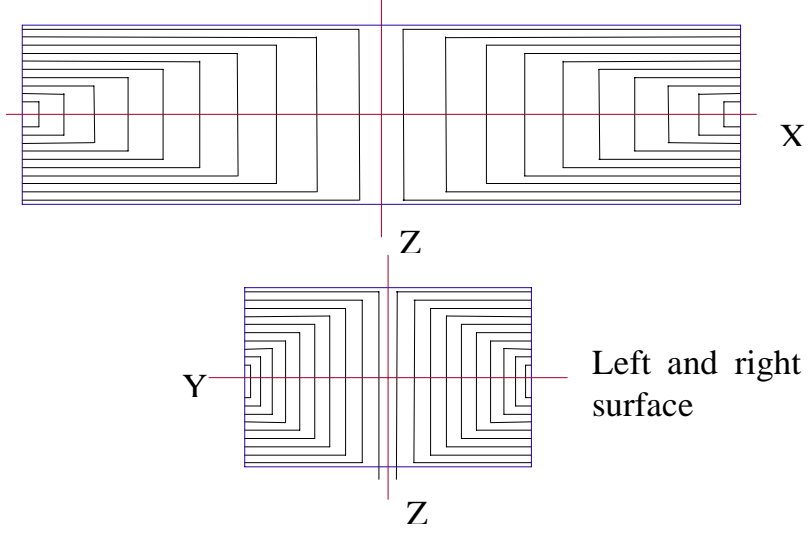

Figure 2: Conductor location of Panofsky quadrupole.

The size of the Panofsky quadrupole used in the ring is $4.4 \mathrm{~cm}$ long, $13.4 \mathrm{~cm}$ wide, and $5.6 \mathrm{~cm}$ high. The design with a 10-loop structure, given in Figure 2, uses $\alpha=-2.14$, $\beta=-2.0$. This design retains good field quality, shown in Table 1, and small inductance, as we will see later.

\subsection{External Connections}

For the final Panofsky quadrupole to be used in UMER, each of the 4 quadrants will be in series with an external $50 \Omega$ resistor and driven by a separate pulse generator. A common dc power supply will be used to power the 4 pulse generators and ensure equal pulse voltage.

For the 10 loop design in section 2.2, a current of $28.2 \mathrm{~A}$ in each conductor is needed to produce an integrated gradient of 28 Gauss. The required generator voltage is $1.4 \mathrm{kV}$. The quadrupole is on for $20 \mu \mathrm{s}$, which corresponds to 100 revolutions in the ring. If the ring operates in $60 \mathrm{~Hz}$, the duty factor is as small as $1.2 \times 10^{-3}$. This gives a total average power of $156 \mathrm{w}$, or $47 \mathrm{w}$ per resistor. The inductance of each quadrant is about $3 \mu \mathrm{H}$ (see section 3.2 for detail), and gives a time constant of 30ns.

The mechanical part of the design is not finalized yet. Both the wire method and a printed circuit are being explored.

Table 1: The deviation of integral gradient at different radius and angle. The pipe radius is $\mathrm{R}=2.5 \mathrm{~cm}$.

\begin{tabular}{|c|c|c|c|c|}
\hline & $0^{\circ}$ & $30^{\circ}$ & $60^{\circ}$ & $90^{\circ}$ \\
\hline $0.2 \mathrm{R}$ & $-0.02 \%$ & $-0.02 \%$ & $0.0 \%$ & $0.03 \%$ \\
\hline $0.4 \mathrm{R}$ & $-0.03 \%$ & $-0.11 \%$ & $0.0 \%$ & $0.19 \%$ \\
\hline $0.7 \mathrm{R}$ & $0.50 \%$ & $-0.63 \%$ & $-0.58 \%$ & $-0.59 \%$ \\
\hline
\end{tabular}

\section{TEST AND SIMULATION}

\subsection{Prototype Field Measurement}

A 2:1 scaled prototype of 16 loops (Figure 3) has been built to measure the magnetic field. (The 10-loop design was done after this measurement). A $1.67 \mathrm{MHz}, 30 \mathrm{~V}$ sinusoidal signal is used to drive the prototype quadrupole, and a small coil of 20 turns is put into the quadrupole to get the induced voltage. The magnetic field is then calculated from the induced voltage. The magnetic field measurement agrees very well with the MAG-PC calculation, which is shown in Figure 4.

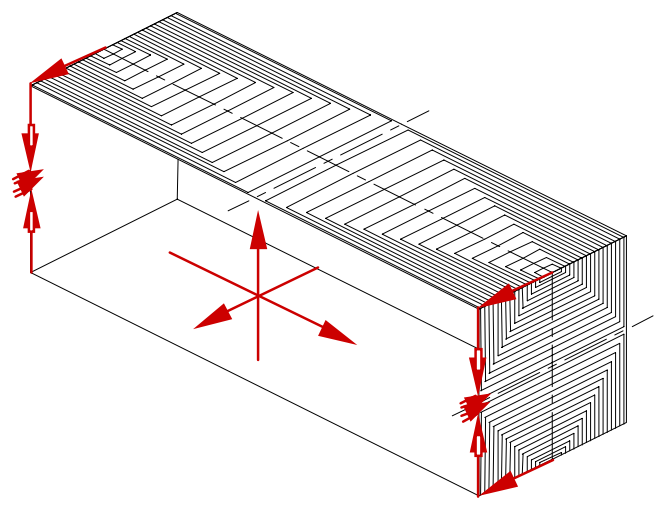

Figure 3: The prototype of Panofsky quadrupole. 


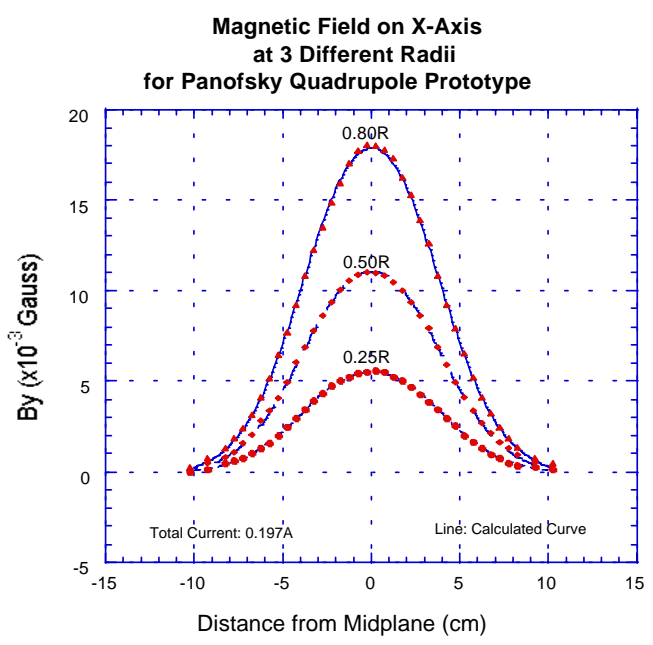

Figure 4: Magnetic field measurement (markers) and calculation (curve).

\subsection{Inductance Measurement}

Two 1:1 models of the 10-loop design have been built to measure the inductance and check the interaction between them. One is a little bit larger than the other in the size. The inductance of each quadrant is $2.5 \mu \mathrm{H}$ and $3.3 \mu \mathrm{H}$ respectively for the two models.

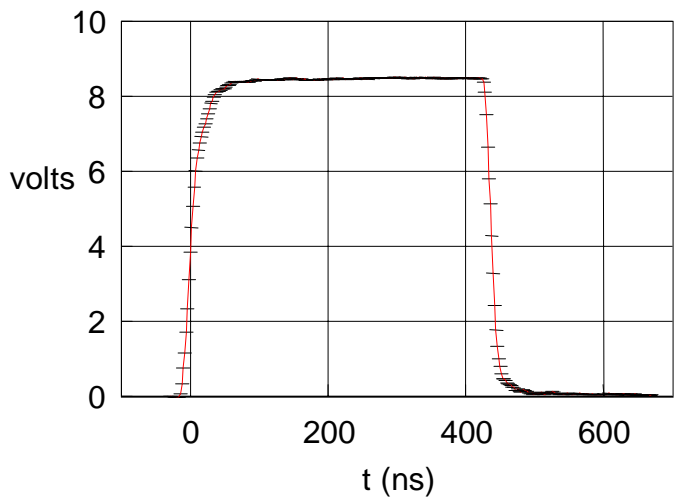

Figure 5: Circuit response of the Panofsky quadrupole model.

In the circuit response measurement, each quadrant is in series with a nominal $22 \Omega$ resistor to guarantee equal current in each quadrant. The 4 quadrants are then in parallel. This arrangement is then in series with a $46 \Omega$ resistor and driven by a rectangular pulse with a time constant about $10 \mathrm{~ns}$. Figure 5 is the signal picked up from the $46 \Omega$ resistor. This signal exhibits fast enough rise time and also fall time.

In order to check the interaction between the quadrupoles, two relative locations have been tested: one is when the two models are far away, the other is when the small model is overlapped with the large one a little bit, according to their relative position in Figure 1. The large one is driven by an 80ns rectangular pulse while the small one is driven by the same pulse but with 100ns delay. The output waveform has no detectable difference between the two cases. This shows that the mutual inductance is not a serious problem. However, these measurements will be repeated in the final arrangement.

In the future, maybe after the Panofsky quadrupole are built, the rotating coil system is to be used in the measurement of the harmonic components of the DC magnetic field; and the taut wire method is being employed to measure the pulsed magnetic field [7].

\subsection{Effect to beam, by Simulation}

The Panofsky quadrupole has a different field profile than the regular cylindrical quadrupoles designed for UMER. Beam simulation by a particle in cell code, WARP [8], has been done to check this effect on the beam. In Figure 6, the lower curve is the emittance growth in the $\mathrm{x}$ direction with only regular quadrupoles, while the upper curve is after changing 2 regular quadrupoles into Panofsky quadrupoles. The result shows that there is no large difference in emittance growth, and therefore verifies the field linearity of the design. [6]

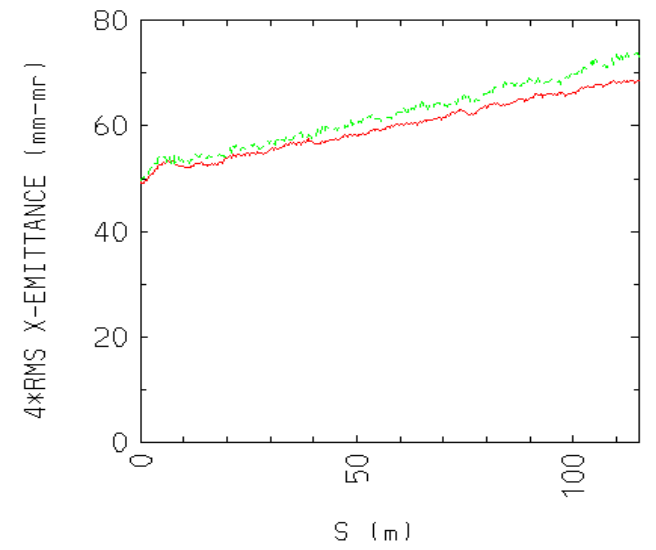

Figure 6. WARP simulation of Panofsky Quadrupole.

\section{SUMMARY}

A pulse Panofsky quadrupole with rectangular aperture is designed. Prototype field measurement and inductance measurement have been done. Simulation has been done to check the linearity. The mechanical construction and pulse generator is under development.

\section{REFERENCE}

[1] M. Reiser, et al., "The Maryland Electron Ring for Investigating Space-Charge Dominated Beams in a Circular FODO System," in these proceedings.

[2] J.G. Wang, et al., Nucl. Instrum. Methods Phys. Res. A. (1998), vol. 415, no. 1-2, P.422-7.

[3] D. Kehne, et al., "The $10 \mathrm{keV}$ Injector for the University of Maryland Electron Ring Project," in these proceedings.

[4] M. Venturini, Electron Ring Project Technical Notes, Nov, 1996.

[5] M. Venturini, Electron Ring Project Technical Notes, July, 1995.

[6] R. Kishek, et al., "Simulations of Collective Effects in the SpaceCharge-Dominated Beam of the University of Maryland Electron Ring," in these proceedings.

[7] W.W. Zhang, et al., "Magnetic Field Measurement of Printed-Circuit Quadrupoles and Dipoles," in these proceedings.

[8] D. P. Grote, et. al., Fus. Eng. \& Des. 32-33, 193-200 (1996). 